\title{
Nonlinear Torsional Vibrations of a Rotating Shaft System
}

with a Magnet Coupling*

\author{
By Hiroyuki KOJIMA** and Kosuke NAGAYA***
}

In this paper, the nonlinear torsional vibrations of a rotating shaft system having a disk and a magnet coupling consisting of rareearth magnets are investigated analytically. The driver of the magnet coupling is excited by sinusoidal motion, and the disk is subjected to constant load torque. The transmission torque of the magnet coupling is represented by a sine function of relative angle between the driver and the follower. The governing partial equations are solved by the harmonic balance method. Besides the harmonic vibration, the onesecond order and the one-third order subharmonic vibrations are obtained. Numerical calculations are carried out by the Newton-Rapson method, and the influences of the load torque on the nonlinear torsional vibrations are investigated.

Key Words: Vibration, Magnet Coupling, Rotating Shaft, Nonlinearity, Torsional Vibration, Rare-Earth Magnet.

\section{Introduction}

Rare-earth magnets possess more super strong magnetic energy than magnets of other materials. The magnet coupling which is operated by the attracting force and the repelling one acting between the rare-earth magnets has several advantages such as the non-contact transmission of torque through partion wall and the effective vibration isolation. Studies on the torsional magnetic stiffness of the magnet coupling have been reported (1), (2), but the dynamic characteristics have not been investigated yet. In this paper, the forced torsional vibrations of a rotating shaft system with a magnet coupling and a disk are investigated analytically in consideration of both the nonlinearity and the unsymmetricity of the magnetic torsional restoring force. The driver of the magnet coupling is excited by sinusoidal motion, and the disk is subjected to constant load torque. In the analysis, first, the transmission torque of the magnet coupling is represented as sine function by using the theoretical and experimental results of the previous report, and the equations of motion of the system are derived. Then, the forced torsional vibration responses are obtained by applying the harmonic balance method in consideration of the onesecond order and the one-third order subharmonics as well as the fundamental one. Furthermore, the numerical calculations are carried out by the Newton-Rapson meth$o d$, and the influences of the load torque on the nonlinear torsional vibrations are investigated.

* Received 6th december, 1982

** Research Associate, Faculty of Engineering, Gunma University, 1-5-1 Tenjin-cho, Kiryu, Gunma 376 *** Professor, The same as the above.

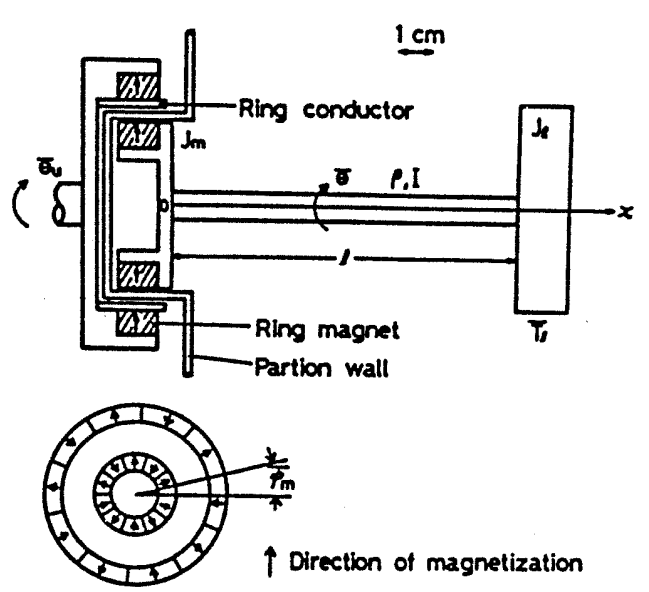

Fig.1 Rotating shaft with a magnet coupling and a disk

\section{Equations of Motion and Boundary Conditions}

Figure 1 shows a rotating shaft system driven by a magnetic coupling. The torque of the driver is transmitted to the follower without mechanical contacts. The system is sujected to the damping force. This damping force is generated by the eddy current caused by the relative motion between the ring conductor attached on the driver and the ring rare-earth magnets set on the follower. The driver is excited by sinusoidal motion $\bar{\theta}_{u}$, and the rotating shaft is connected to the follower. A disk is attached to the rotating shaft, and this disk is subjected to the constant load torque $\bar{T}_{l}$. The directions of magnetization of rare-earth magnets are illustrated in Fig.1.

The transmission torque $\bar{T}$ is generated by the attractive force and the repelling one acting between the magnets of both sides, and the torque is a function of the relative angle $\bar{\psi}_{m}$ between the driver and 


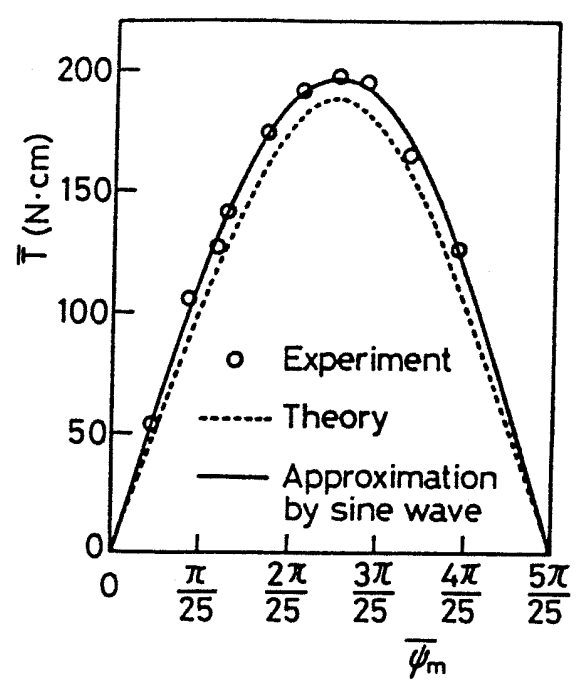

Fig. 2 Experimental and theoretical results of magnetic transmission torque by Weissmann et al.

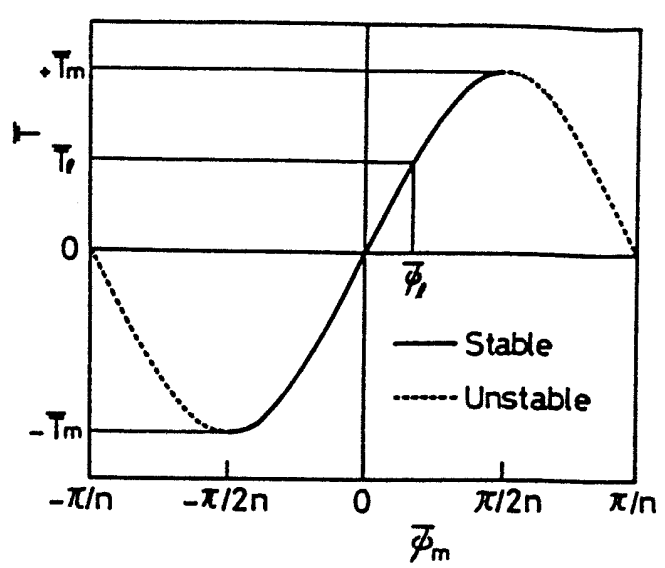

Fig. 3 Magnetic transmission torque when the number of magnetic poles is $2 \mathfrak{n}$

the follwer. Figure 2 shows the experimental and theoretical results of the magnetic transmission torque by Weissmann et al ( $^{(1)}$ who used the rare-earth magnets $\left[\mathrm{B}_{r}=0.9 \mathrm{~T}\right.$, $\mathrm{H}_{c}=700 \mathrm{kA} / \mathrm{m},(\mathrm{BH})_{\max }=159 \mathrm{~kJ} / \mathrm{m}$, the magnets of the driver: $\phi 61.2 \mathrm{~mm} / 75.8 \mathrm{~mm} \times 12 \mathrm{~mm}$, the magnets of the follower: $\phi 33.4 \mathrm{~mm} / 47.7 \mathrm{~mm} \times 12 \mathrm{~mm}$ ]. It is seen that the expeimental results agree well with the theoretical ones, and the magnetic transmission torque can be represented as sine function in practice. Figure 3 shows the magnetic transmission torque of the magnet coupling with $2 \mathrm{n}$ magnetic poles. In this figure, the solid line represents the static stable portion, the dotted line is the static unstable portion, $\bar{T}_{l}$ is the load torque, and $\bar{\phi}_{l}$ is the load angle. The transmission torque $\bar{T}$
is written as

$$
\bar{T}=\bar{T}_{m} \sin n \bar{\psi}_{m}
$$

whera $\overline{\mathrm{T}}_{m}$ is the maximum value of the transmission torque. On the other hand, the equation of motion of the rotating shaft is expressed using the notations in Fig.I as

$$
\frac{\partial^{2} \theta}{\partial t^{2}}=\frac{G}{\rho} \frac{\partial^{2} \theta}{\partial x^{2}}
$$

where $\theta(=n \bar{\theta})$ is the angular dispacement of the rotating shaft, $G$ is the modulus of shear of the shaft, $\rho$ is the mass density. Assuming that the load angle $\bar{f}$ is constant, we can expand $T$ into Tailor Series in the vicinity of $\psi_{q}$, and three members of this series are utilized in the following analysis. As the result, the boundary conditions are
obtained as follows:

$$
\begin{aligned}
& x=0: G I \frac{\partial \theta}{\partial x}=J_{m} \frac{\partial^{2} \theta}{\partial t^{2}}-\left\{T_{t}+\left(T_{m} \cos \psi_{t}\right)\left(\theta_{m}-\frac{1}{2} \theta_{m}^{2} \tan \psi_{l}-\frac{1}{6} \theta_{m}^{3}\right)\right\}-C_{m} \frac{d \theta_{m}}{d t} \\
& x=l: G I \frac{\partial \theta}{\partial x}=-J_{l} \frac{\partial^{2} \theta}{\partial t^{2}}-T_{l}
\end{aligned}
$$

where

$$
\left.\begin{array}{l}
\theta_{m}=\psi_{m}-\psi_{l}=\theta_{u}-(\theta)_{x-0}-\psi_{t}, \theta_{u}=\phi_{u} \cos \omega t, \theta_{m}=n \bar{\theta}_{m} \\
\theta_{u}=n \bar{\theta}_{u}, \psi_{m}=n \bar{\psi}_{m}, \psi_{l}=n \bar{\psi}_{l}, T_{t}=n \bar{T}_{l}, T_{m}=n \bar{T}_{m}
\end{array}\right\}
$$

and where $\bar{\theta}_{m}$ is the relative displacement from the load angle $\bar{\psi}_{k}$, I is the polar moment of area, $C_{m}$ is the coefficient of the damping force term for the eddy current. The quantities such as the angular displacement and the torque are normalized as Eqs. (5) by
using the number of magnetic poles.

\section{Solutions of Equations of Motion}

In consideration of the one-second order and one-third order subharmonics as well as the fundamental one, we assume the solutions of the steady- state vibrations of the angular displacement $\theta, \theta_{m}$ as follows:

$$
\left.\begin{array}{l}
\theta=\phi_{s}+\sum_{j=1}^{3}\left(\phi_{j} \cos n_{j} \omega t+\bar{\phi}_{j} \sin n_{j} \omega t\right) \\
\theta_{m}=\psi_{s}+\sum_{j=1}^{3}\left(\phi_{j} \cos n_{j} \omega t+\bar{\phi}_{j} \sin n_{j} \omega t\right)
\end{array}\right\}
$$


where

$$
n_{1}=1, n_{2}=1 / 2, n_{3}=1 / 3
$$

Substituting Eq.(6) into Eq.(2) gives the following expressions:

$$
\left.\begin{array}{l}
\phi_{3}=A_{0}+A_{1} x, \phi_{i}=B_{1 j} \cos n_{j} \lambda x+B_{2 j} \sin n_{j} \lambda x \\
\bar{\phi}_{j}=\bar{B}_{1 j} \cos n_{j} \lambda x+\bar{B}_{2 j} \sin n_{j} \lambda x
\end{array}\right\}
$$

where

$$
\lambda^{2}=\rho \omega^{2} / G
$$

The notaions $A_{0}, A_{1}, B_{1 j}, B_{2 j}, \bar{B}_{1 j}, \bar{B}_{2 j}$ in Eq. (8) are unknown constants to be determined by the boundary conditions. By substituting Eqs. (6)-(9) into Eq. (4), the unknown cons-
tants can be expressed as

$$
A_{0}=Q_{1}+\frac{T_{i} l}{G I}, A_{1}=-\frac{T_{l}}{G I}, B_{1 j}=H_{1}, Q_{3}, B_{2 j}=H_{2 j} Q_{3}, \bar{B}_{1 j}=H_{1 j} \bar{Q}_{j,}, \bar{B}_{2 j}=H_{2 j}, \bar{Q},
$$

where

$$
\begin{aligned}
& H_{1 j}=\cos n_{j} \lambda l-n_{j} x_{1} \lambda l \sin n_{j} \lambda l, H_{2 j}=\sin n_{j} \lambda l+n_{j} x_{l} \lambda l \cos n_{j} \lambda l \\
& Q_{s}=\left(\phi_{s}\right)_{x=l,}, Q_{J}=(\phi)_{x=l}, \bar{Q}_{J}=(\bar{\phi})_{x=l,}, x_{l}=J_{l} / J_{s}, J_{s}=\rho l I \quad \ldots .
\end{aligned}
$$

and $\mathrm{J}_{\ell}$ is the moment of inertia of the disk, $J_{s}$ is that of the rotating shaft. By substituting Eqs. (6)-(12) into the boundary condition (3) and applying the harmonic balance method, the nonlinear simultaneous equations determining the amplitude components shown in Eq. (6) are derived as follows:

$$
\begin{aligned}
& W_{i}-g_{s}=0 \ldots \ldots \ldots \ldots \ldots \ldots \ldots \ldots \ldots \ldots \ldots \ldots \ldots \ldots \ldots \ldots \ldots \ldots \ldots \ldots \ldots \ldots \ldots \ldots \\
& \left\{x_{m} n_{j}^{2} H_{1 j}(\lambda l)^{2}+n_{j} \lambda l H_{2 j}-x H_{1}, \cos \psi_{l}\right\} W_{j} \\
& \quad-\left\{2 \mu \eta n_{j} H_{1 j}\left(\lambda_{1} l\right) \sqrt{x x_{m} \cos \psi_{l}}\right\} \bar{W}_{j}+x H_{1 j} g_{j} \cos \psi_{l} \\
& \quad=\left\{x_{m} n_{j}^{2} H_{1 j}(\lambda l)^{2}+n_{j} \lambda l H_{2 j}\right\} \delta_{1 j} \\
& 2 \mu \eta n_{j} H_{1 j}\left(\lambda_{1} l\right) \sqrt{x x_{m} \cos \phi_{l}} W_{j}+\left\{x_{m} n_{j}^{2} H_{1 j}(\lambda l)^{2}\right. \\
& \left.\quad+n_{j} \lambda l H_{2 j}-x H_{1 j} \cos \psi_{l}\right\} \bar{W}_{j}+x H_{1 j} \bar{g}_{j} \cos \psi_{l}=0 \\
& (j=1,2,3)
\end{aligned}
$$

In the above equations, the unknown quantities are the magnification factors of the follower of the magnet coupling, $\delta, \delta$ is the Kronecker delta, and

$$
\begin{aligned}
& g_{4}=\varepsilon_{2}\left\{W_{3}^{2}+\frac{1}{2}\left(W_{1}^{2}+\bar{W}_{1}^{2}+W_{2}^{2}+\bar{W}_{2}^{2}+W_{3}^{2}+\bar{W}_{3}^{2}\right)\right\}+\varepsilon_{3}\left\{W_{3}^{3}+\frac{3}{2} W_{3}\left(W_{1}^{2}+\bar{W}_{1}^{2}+W_{2}^{2}\right.\right. \\
& \left.\left.+\bar{W}_{2}^{2}+W_{3}^{2}+\bar{W}_{3}^{2}\right)+\frac{3}{2} \bar{W}_{1} W_{2} \bar{W}_{2}+\frac{3}{4} W_{1}\left(W_{2}^{2}-\bar{W}_{2}^{2}\right)\right\} \\
& g_{1}=\varepsilon_{2}\left\{\frac{1}{2}\left(W_{2}^{2}-\bar{W}_{2}^{2}\right)+2 W_{1} W_{1}\right\}+\varepsilon_{3}\left\{3 W_{s}^{2} W_{1}+\frac{1}{4} W_{3}^{3}+\frac{3}{4}\left(W_{1}^{3}+W_{1} \bar{W}_{1}^{2}-W_{3} \bar{W}_{3}^{2}\right)\right. \\
& \left.+\frac{3}{2} W_{1}\left(W_{2}^{2}-\bar{W}_{2}^{2}\right)+\frac{3}{2} W_{1}\left(W_{2}^{2}+\bar{W}_{2}^{2}+W_{3}^{2}+\bar{W}_{3}^{2}\right)\right\} \\
& g_{2}=\varepsilon_{2}\left(2 W_{1} W_{2}+W_{1} W_{2}+W_{1} \bar{W}_{2}\right)+\varepsilon_{3}\left\{3 W_{2}^{2} W_{2}+\frac{3}{4} W_{2}\left(W_{2}^{2}+\bar{W}_{2}^{2}\right)\right. \\
& \left.+\frac{3}{2} W_{2}\left(W_{1}^{2}+\bar{W}_{1}^{2}+W_{3}^{2}+\bar{W}_{3}^{2}\right)+3 W_{1}\left(W_{1} W_{2}+\bar{W}_{1} \bar{W}_{2}\right)\right\} \\
& g_{3}=2 \varepsilon_{2} W_{3} W_{3}+\varepsilon_{3}\left\{3 W_{3}^{2} W_{3}+\frac{3}{4}\left(W_{3}^{3}+W_{3} \bar{W}_{3}^{2}+W_{1} W_{3}^{2}-W_{1} \bar{W}_{3}^{2}\right)\right. \\
& \left.+\frac{3}{2}\left(W_{1}^{2} W_{3}+\bar{W}_{1}^{2} W_{3}+\bar{W}_{1} W_{3} \bar{W}_{3}+W_{2}^{2} W_{3}+\bar{W}_{2}^{2} W_{3}\right)\right\} \\
& \bar{g}_{1}=\varepsilon_{2}\left(W_{2} \bar{W}_{2}+2 W_{1} \bar{W}_{1}\right)+\varepsilon_{3}\left\{3 W_{3}\left(W_{3} \bar{W}_{1}+W_{2} \bar{W}_{2}\right)-\frac{1}{4} \bar{W}_{3}^{3}\right. \\
& \left.+\frac{3}{4}\left(\bar{W}_{1}^{3}+W_{1}^{2} \bar{W}_{1}+W_{3}^{2} W_{3}\right)+\frac{3}{2} \bar{W}_{1}\left(W_{2}^{2}+\bar{W}_{2}^{2}+W_{3}^{2}+\bar{W}_{3}^{2}\right)\right\} \\
& \bar{g}_{2}=\varepsilon_{2}\left(2 W_{1} \bar{W}_{2}-W_{1} \bar{W}_{2}+\bar{W}_{1} W_{2}\right)+\varepsilon_{3}\left\{3 W_{1}\left(W_{1} \bar{W}_{2}+\bar{W}_{1} W_{2}-W_{1} \bar{W}_{2}\right)\right. \\
& \left.+\frac{3}{4} \bar{W}_{2}\left(W_{2}^{2}+\bar{W}_{2}^{2}\right)+\frac{3}{2} \bar{W}_{2}\left(W_{1}^{2}+\bar{W}_{1}^{2}+W_{3}^{2}+\bar{W}_{3}^{2}\right)\right\} \\
& \bar{g}_{3}=2 \varepsilon_{2} W_{2} \bar{W}_{3}+\varepsilon_{3}\left\{3 W_{2}^{2} \bar{W}_{3}+\frac{3}{4}\left(\bar{W}_{3}^{3}+W_{3}^{2} \bar{W}_{3}+\bar{W}_{1} W_{3}^{2}-\bar{W}_{1} \bar{W}_{3}^{2}\right)\right. \\
& \left.+\frac{3}{2} \bar{W}_{3}\left(W_{1}^{2}+\bar{W}_{1}^{2}-W_{1} W_{3}+W_{2}^{2}+\bar{W}_{2}^{2}\right)\right\}
\end{aligned}
$$




$$
\left.\begin{array}{l}
W_{s}=\frac{\phi_{s}}{\phi_{s}}, W_{s}=\frac{\phi_{j}}{\phi_{u}}, \quad \bar{W}_{s}=\frac{\bar{\phi}_{3}}{\phi_{u}}, \varepsilon_{2}=\frac{1}{2} \phi_{u} \tan \psi_{l}, \varepsilon_{3}=\frac{1}{6} \phi_{u,}^{2} \lambda l=\eta\left(\lambda_{1} l\right), \eta=\frac{\omega}{\omega_{1}} \\
\omega_{1}=\frac{\lambda_{1} l}{l} \sqrt{\frac{G}{\rho}}, x_{m}=\frac{J_{m}}{J_{s}}, \quad x=\frac{T_{m}}{k_{s}}, k_{z}=\frac{G l}{l}, \mu=\frac{C_{m}}{2 \sqrt{J_{m} T_{m} \cos \psi_{l}}}
\end{array}\right\}
$$

and $\omega_{1}, \lambda_{1} l$ are the natural frequency and the eigenvalue for the first mode of the system obtained by neglecting the nonlinearity, $\varepsilon_{2}, \varepsilon_{3}$ are the coefficients of the nonlinear terms for the magnetic torsional restoring force and $\eta$ is the frequency ratio. Then, the nonlinear simultaneous equations (13), (14) can be solved by using the Newton-Raphson method. On the other hand, the magnification factors of the disk are obtained as

$$
V_{j}=\left(\delta_{1, j}-W_{j}\right) / H_{1}, \quad \bar{V}_{j}=-\bar{W}_{j} / H_{1 j}
$$

where

$$
V_{j}=Q_{j} / \phi_{u}, \bar{V}_{j}=\bar{Q}_{j} / \phi_{u}
$$

By putting $\phi_{u}=0, C_{m}=0$ in Eqs. (3) and (5), the solutions for the free vibrations of the system may be obtained. We assume the solutions as

$$
\theta=\phi_{i}^{*}+\phi^{*} \cos \omega t, \theta_{m}=\phi_{i}^{*}+\psi^{*} \cos \omega t
$$

Substituting Eqs.(21) into Eqs.(2)-(4) gives the equations determining the amplitude.

$$
\begin{gathered}
W_{i}^{*}-\varepsilon_{2}\left(W_{i}^{* 2}+\frac{1}{2} W^{* 2}\right)-\varepsilon_{3}\left(W_{i}^{* 3}+\frac{3}{2} W^{*} W^{* 2}\right)=0 \\
\left\{x_{m} H_{11}(\lambda l)^{2}+\lambda l H_{21}-x H_{11} \cos \psi_{l}\right\} W^{*}+x H_{11} \cos \psi_{l}\left\{2 \varepsilon_{2} W_{i}^{*} W^{*}\right\} \\
\left.\quad+\varepsilon_{3}\left(\frac{3}{4} W^{* 3}+3 W_{i}^{* 2} W^{*}\right)\right\}=0 \\
V^{*}=-W^{*} / H_{11}
\end{gathered}
$$

where

$$
W^{*}=\psi^{*} / \phi_{u}, W=\psi^{*} / \phi_{u}, V^{*}=\left(\phi^{*}\right)_{x=1} / \phi_{u}
$$

In Eqs. (22)-(24), the amplitude and the shifting amount of the vibration center are expressed as dimensionless quantities obtained by using the exciting amplitude $\phi_{u}$ used in the forced vibrations. Then, the magnification factor $V_{\ell}$ of the disk neglecting the nonlinearity of the magnetic torsional restoring force is written as

$$
V_{l} \doteq\left\{\left(R_{\mathrm{t}}^{2}+R_{2}^{2}\right) /\left(R_{3}^{2}+R_{4}^{2}\right)\right\}^{1 / 2}
$$

where

$$
\begin{aligned}
& R_{1}=x \cos \psi_{l}, R_{2}=2 \mu \eta\left(\lambda_{1} l\right) \sqrt{x x_{m} \cos \psi_{l}} \\
& R_{3}=\lambda l H_{21}+x_{m}(\lambda l)^{2} H_{11}-x H_{11} \cos \psi_{l} \\
& R_{4}=2 \mu \eta\left(\lambda_{l} l\right) H_{41} \sqrt{x x_{m} \cos \psi_{l}}
\end{aligned}
$$

Table 1 Eigenvalue $\lambda_{n} l$

\begin{tabular}{l|l|l|l}
\hline \multirow{2}{*}{$\mathrm{n}$} & \multicolumn{3}{|c}{$\varphi_{\ell}(\mathrm{rad})$} \\
\cline { 2 - 4 } & \multicolumn{1}{|c}{0} & \multicolumn{1}{|c}{0.4} & \multicolumn{1}{|c}{0.8} \\
\hline 1 & 0.00664 & 0.00638 & 0.00556 \\
2 & 0.08581 & 0.08576 & 0.08561 \\
3 & 3.1439 & 3.1439 & 3.1439 \\
4 & 6.2843 & 6.2843 & 6.2843 \\
\hline
\end{tabular}

4. Numerical Calculations and Considerations

In the following numerical calculations, the dimensions of the magnet coup1ing, the rotating shaft and the disk are the same as those of the model shown in Fig.1, and the results of the magnetic transmission torque in Fig. 2 by Weissmann et al. are utilized. By assuming that the materials of the rotating shaft, the disk and the magnet couling are steel, the following values are obtained.

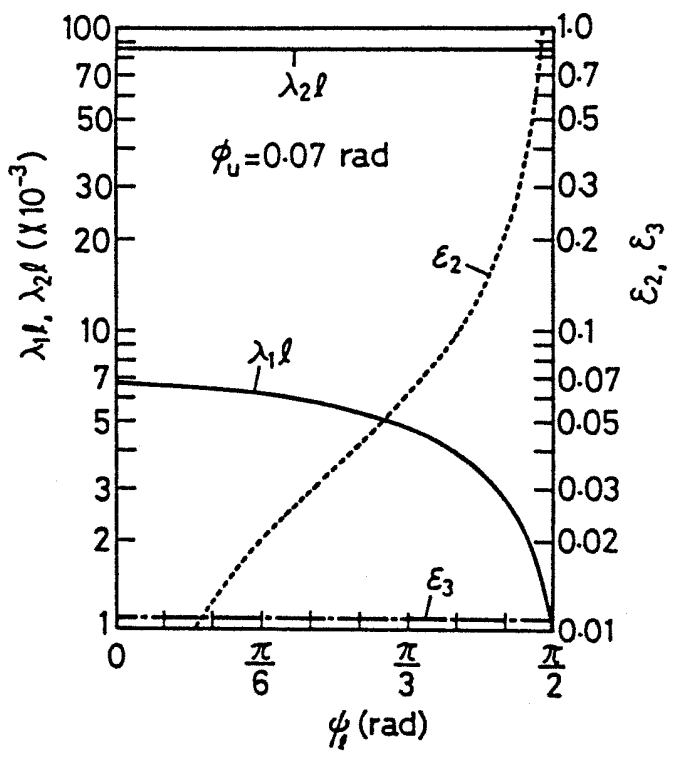

Fig. 4 Eigenvalue and coeffients of nonlinear terms versus load angule 


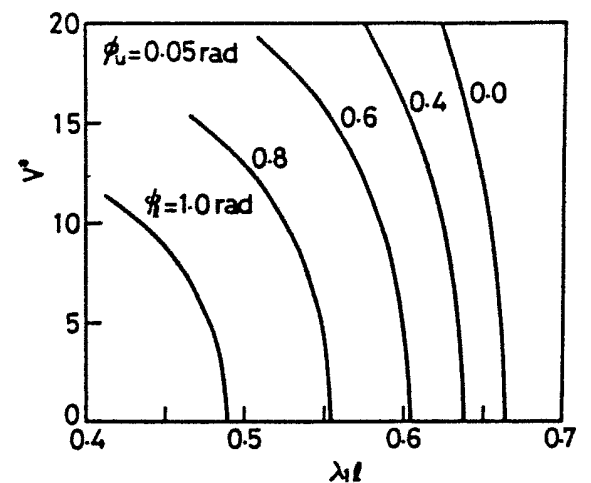

Fig. 5 Effects of load angle on free vibration

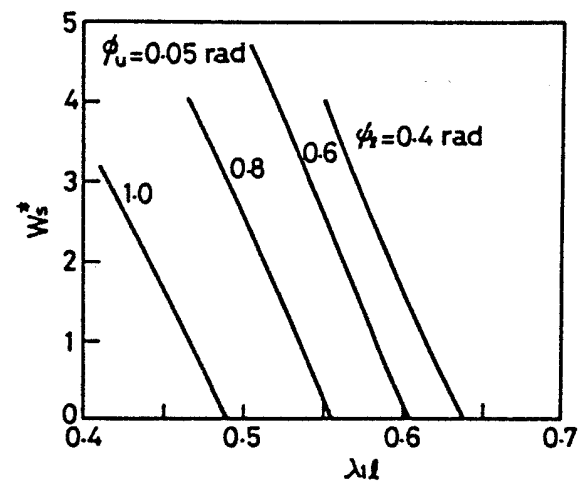

Fig. 6 Shifting amount of vibration center under free vibration

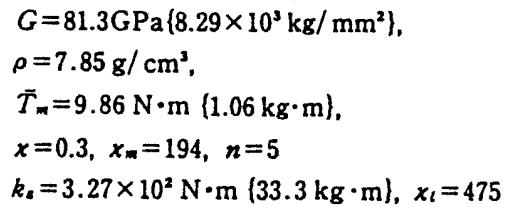

where the mass density of the rare-earth magnets is $8.2 \mathrm{~g} / \mathrm{cm}^{3}(1)$. Table 1 show the eigenvalues of the system linearized about the load torque $\psi$. $\lambda_{1} l$ corresponds to the natural frequency by the magnetic torsional restoring force, $\lambda_{2} l$ the one by the torsional stiffness of the rotating shaft and the moments of the inertia of the follower and the disk, $\lambda_{3} \ell, \lambda_{4} \ell$ the ones by the rotating shaft only. It is shown that $\lambda_{1} \ell$ is considerably smaller than $\lambda_{2} l$. Thus, it is seen that the natural frequency of the system can be designed to be extremely small by use of the magnet coupling, and that the vibration isolation may be accomplished effectively. Figure 4 shows the eigenvalues and the coefficients of the nonlinear terms $\varepsilon_{2}$, $\varepsilon_{3}$ against the load angle $\psi_{q}$. The eigenvalue $\lambda_{1} \ell$ decreases with an increasing value of $\psi_{\ell}$, but $\lambda_{2} \ell$ is approximately constant. As $\psi_{l}$ increases, $\varepsilon_{2}$ becomes larger, and the magnetic torsional restoring force exhibits a more remarkable unsymetrical character. Furthermore,

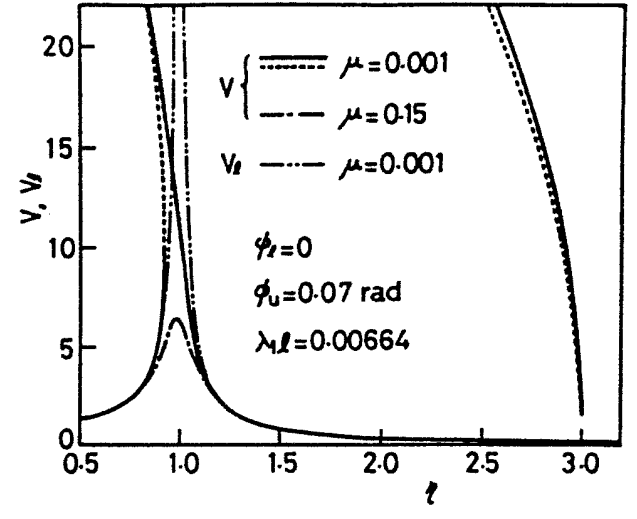

Fig.7 Torsional forced vibration response

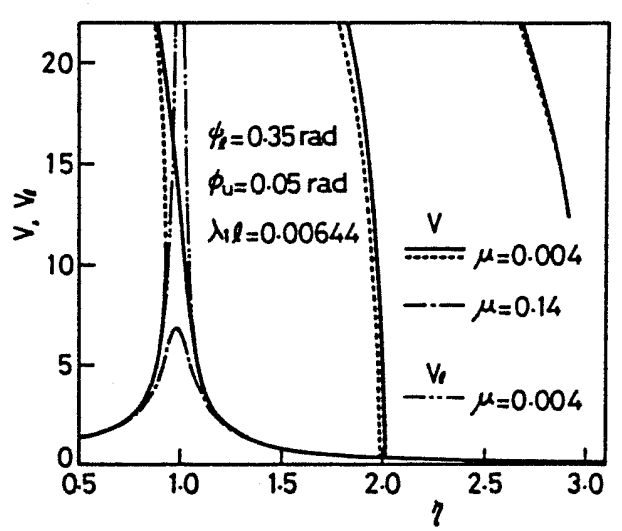

Fig.8 Torsional forced vibration response

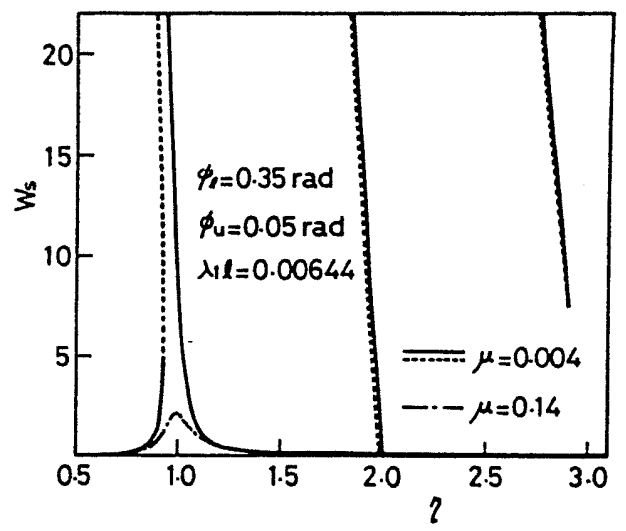

Fig. 9 Shifting amount of vibration center under torsional forced vibration

$\varepsilon_{3}$ is constant.

We define the dimensionless magnification factor by use of all the components in Eqs. (6) as

$$
V=\left[\sum_{j=1}^{3}\left(V_{j}^{2}+\bar{V}_{j}^{2}\right)\right]^{1 / 2}
$$

where $\mathrm{V}$ represents the magnification factor at the disk. In the following numerical calculations, the magnification fact- 
ors at the disk are obtained, and the shifting amount of the vibration center of the relative angle between the driver and the follower is calculated.

Figure 5 and 6 show the free vibrations and the shifting amount of the vibration center versus the load angle. The free vibrations show a more remarkable softening spring character with an increasing value of the load angle. Then, the shifting amounts of the vibration center are approximately proportional to the decreasing amount of the eigenvalue. Figure 7 and 8 depict the forced torsional vibration responses. In each figure, a solid line and a one-dot chain line indicate the stable solution, a broken line the unstable one, and a two-dot line the linear one in case of ignoring the nonlinearity. In the present paper, the determination of the stability has been carried out by adopting the argument that the point having a vertical tangent to the response curve is a limiting point, and has not been investigated theoretically. Fugure 7 shows the response curves when the load angle is zero. The nonlinear response curve indicates a remarkable softening character, and differs considerably from the linear response curve. As the damping force increases, the amplitude of the fundamental harmonic resonance is reduced to a small value. When the system is subjected to a small damping force, only onethird order subharmonic vibration occurs, for the magnetic restoring force has no unsymmetrical character. Figure 8 depicts the response curves in case of $\phi_{\ell}=0.35$ rad. In this case, the one-second order and the one-third order subharmonics occur, for the magnetic torsional restoring force has an unsymmetrical character. The shifting amounts of the vibration center are shown in Fig.9. It is observed that the shifting amounts of the vibration center become remarkably large at the one-second order and the one-third order subharmonic resonances as well as the fundamental one.

\section{Conculsions}

In this paper, the torsional forced vibrations of the rotating shaft system driven by a magnet coupling are investigated theoretically with consideration of the one-second order and the one-third order subharmonics as well as the fundamental one. The magnetic transmission torque of the magnet coupling is represented as sine function of relative angle between the driver and the follower. A magnet coupling and a disk are attached to both ends of the rotating shaft. Furthermore, the driver of the magnet coupling is excited by sinusoidal motion, and the disk is subjected to constant load torque. The results may be summarized as follows:

(1) By using the magnet coupling, the natural frequency of the system can be designed to be remarkably small. Therefore, the vibration isolation may be carried out effectively.

(2) As the load torque increases, the eigenvalue for the first mode decreases, and the magnetic torsional restoring force shows a more remarkable unsymmetrical character. When the load torque is zero, the unsymmetrical character disappears.

(3) The torsional forced vibration responses show a remarkable softening character. When the system is subjected to a small damping force, the one-second order and one-third order subharmonics occur. When the load torque is zero, only one-third order subharmonic is generated.

\section{Acknowledgements}

The authors would like to express their deep appreciation to the Emeritus Professor Hideo Saito of Tohoku University and the Emeritus Professor Izumo Yamakawa of Gunma University for their consistent guidance and encouragement. They also wish to thank Mr. Sigeo Ikai of Gunma University for his cooperation in this work.

\section{References}

(1) Weissmann, D., et al., Proc. 3rd Int. Workshop R. E-Co Mag. App1., 6(1978), 325.

(2) Fellows, C, J., Chartered Mech. Eng., 26-6(1979), 79 . 